CHPRC-00573

Revision 0

\title{
100-KE Reactor Core Removal Project Alternative Analysis Workshop Report
}

Prepared for the U.S. Department of Energy Assistant Secretary for Environmental Management

Contractor for the U.S. Department of Energy under Contract DE-AC06-08RL14788

CH2MHILL

Plateau Remediation Company

P.O. Box 1600

Richland, Washington 99352 
CHPRC-00573

Revision 0

\section{0-KE Reactor Core Removal Project Alternative Analysis Workshop Report}

Document Type: TR

R. A. Harrington

CH2M HILL Plateau Remediation Company

Date Published

January 2010

Prepared for the U.S. Department of Energy

Assistant Secretary for Environmental Management

Contractor for the U.S. Department of Energy

under Contract DE-AC06-08RL14788

\section{CH2MHILL}

Plateau Remediation Company

P.O. Box 1600

Richland, Washington

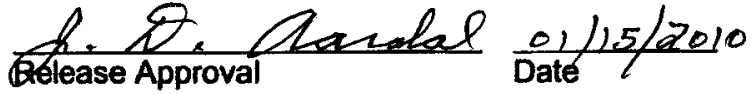

\section{Approved for Public Release; \\ Further Dissemination IJnlimited}




\section{TRADEMARK DISCLAMMER}

Reference herein to any specific commercial product, process,

or service by trade name, trademark, manufacturer, or

otherwise, does not necessarily constitute or imply its

endorsement, recommendation, or favoring by the United

States Government or any agency thereof or its contractors or subcontractors.

This report has been reproduced from the best available copy.

Printed in the United States of America 


\section{0-KE Reactor Core Removal Project}

\section{Alternative Analysis Workshop}

December 15-16, 2009

Requested by: $\quad$ Colburn Kennedy, Project Director CH2M HILL Plateau Remediation Company

Richland, Washington

Facilitated by: Richard A. Harrington, CVS-Life

Christine E. Sumner

CH2M HILL Plateau Remediation Company

Richland, Washington 
CHPRC-00573

Revision 0

\section{TABLE OF CONTENTS}

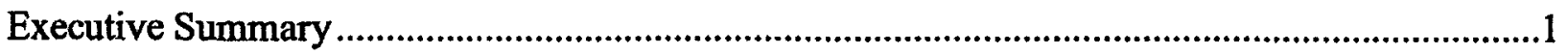

Appendices

A. Path Forward Action Items ................................................................................................

B. Evaluation Matrix, Matrix Conclusions, Criteria, and Advantages/Disadvantages ...................4

C. Session Parking Lot Information Sheets and Presentation Notes............................................13

D. Workshop Agenda, Guidelines/Expectations Opening Remarks and Attendance Roster .......18 
On December 15-16, 2009, a 100-KE Reactor Core Removal Project Alternative Analysis Workshop was conducted at the Washington State University Consolidated Information Center, Room 214. Colburn Kennedy, Project Director, CH2M HILL Plateau Remediation Company (CHPRC) requested the workshop and Richard Harrington provided facilitation. The purpose of the session was to select the preferred Bio Shield Alternative, for integration with the Thermal Shield and Core Removal and develop the path forward to proceed with project delivery. Prior to this workshop, the S.A. Robotics (SAR) Obstruction Removal Alternatives Analysis (565-DLV062) report was issued, for use prior to and throughout the session, to all the team members.

The multidisciplinary team consisted of representatives from 100-KE Project Management, Engineering, Radcon, Nuclear Safety, Fire Protection, Crane/Rigging, SAR Project Engineering, the Department of Energy Richland Field Office, Environmental Protection Agency, Washington State Department of Ecology, Defense Nuclear Facility Safety Board, and Deactivation and Decommission subject matter experts from corporate CH2M HILL and Lucas. Appendix D contains the workshop agenda, guidelines and expectations, opening remarks, and attendance roster going into followed throughout the workshop.

\section{Session Results}

The team was successful in selecting the preferred alternative and developing an eight-point path forward action plan to proceed with conceptual design. Conventional Demolition was selected as the preferred alternative over two other alternatives: Diamond Wire with Options, and Harmonic Delamination with Conventional Demolition. The teams' preferred alternative aligned with the SAR Obstruction Removal Alternative Analysis report conclusion. However, the team identified several Path Forward actions, in Appendix A, which upon completion will solidify and potentially enhance the Conventional Demolition alternative with multiple options and approaches to achieve project delivery.

In brief, the Path Forward was developed to reconsider potential open air demolition areas; characterize to determine if any zircaloy exists, evaluate existing concrete data to determine additional characterization needs, size the new building to accommodate human machine interface and tooling, consider bucket thumb and use of shape-charges in design, and finally to utilize complex-wide and industry explosive demolition lessons learned in the design approach.

Appendix B documents these results from the team's use of Value Engineering process tools entitled Weighted Analysis Alternative Matrix, Matrix Conclusions, Evaluation Criteria, and Alternative Advantages and Disadvantages. These results were further supported with the team's validation of parking-lot information sheets: memories (potential ideas to consider), issues/concerns, and assumptions, contained in Appendix C. Appendix $\mathrm{C}$ also includes the recorded workshop flipchart notes taken from the SAR Alternatives and Project Overview presentations. The SAR workshop presentations, including a 3-D graphic illustration demonstration video have been retained in the CHPRC project file, and were not included in this report due to size limitations.

The workshop concluded with a round robin close-out where each member was engaged for any last minute items and meeting utility. In summary, the team felt the session was value added and looked forward to proceeding with the recommended actions and conceptual design. 


\section{Session Process}

The facilitator opened the session with review of the purpose, safety topic, agenda, and team member introductions. Mr.'s Colburn Kennedy, Kurt Kehler, and Tom Teynor delivered the opening remarks which centered on having a good craft cross section and subject matter experts on the team to define and implement the administrative hazard controls necessary, in this first step, to safely and economically get the core out.

Following the opening remarks, Mark Morton presented the Project Overview Scope and Layout, and Mark Stauder presented the SAR Obstruction Removal Alternatives. The four alternative presentations, with the SAR reference alternatives were 1. Diamond Wire and Options (SAR alternatives 1,2, and 3), 2. Conventional Demolition (SAR alternatives 4 and 8), 3. Water Jet Cutting (SAR alternative 5), and 4. Explosives and Expansive Grout (SAR alternatives 6 and 7).

After each presentation, time was allotted to liquidate all questions, answers, and input to ensure the teams' understanding of each alternative, and how that alternative would meet or exceed design criteria and requirements. Throughout this process any supplemental information, such as memories (i.e., ideas/concepts), enabling assumptions, issues/concerns, and salient alternative points were recorded on flipcharts (a.k.a., parking-lot sheets) for recall. In addition, any item of significant importance was denoted by a "flag-note" symbol $(k)$ for quick visual reference. Following the presentations, the facilitator reviewed the evaluation criteria purpose and a draft set of weighted criteria used to prompt input, revisions, and eventual application.

This process involved two steps: definition and weighting. Following considerable dialogue the team selected and weighed five criterions contained in Appendix B, page 7. At this point, the facilitator led the team into defining advantages and disadvantages of each alternative against each of the five criterions, and areas of significant impact; Appendix B, pages 8-12. Once again, any supplemental information identified by the team was recorded on the parking-lot information sheets. Upon completing the alternative advantages and disadvantages, the facilitator reviewed all inputs and incorporated any of the teams' clarifying inputs and/or new items. For example, the team concluded Alternative 3, Water Jet Cutting, should be removed from further evaluation as it was the most expensive approach, and the pro's would far outweigh the con's. As such, three alternatives were carried over to the next step, weighted analysis alternative.

During the weighted analysis alternative matrix the team evaluated and rated each alternative against each evaluation criteria. The facilitator reviewed the matrix results and solicited the team's final thoughts or conclusions. Several conclusions, such as explosives are a tool and not a complete alternative, were identified and recorded, on page 6 . The team then developed the eight path forward actions (Appendix A), following review and validation of the parking-lot information sheets. The workshop concluded with a round robin close-out to solicit any item not addressed and closing remarks from all in attendance.

\section{Facilitator Comments}

The session went well. Each team member was engaged throughout this process and actively participated and synergized off each other's input to producing these results. Special thanks to Colburn Kennedy, Dave Lowe, and the Bob Norris led SAR project engineering team for their support input and participation prior to and throughout this session. 
CHPRC-00573

Revision 0

\section{APPENDIX A}

\section{PATH FORWARD ACTION ITEMS}

\begin{tabular}{|c|c|c|c|}
\hline \multicolumn{4}{|c|}{ PATH FORWARD ACTION ITEMS } \\
\hline & What & Who & When \\
\hline 1 & Reconsider potential areas for open air demo & $\begin{array}{l}\text { Colburn Kennedy } \\
\text { Darren Boone }\end{array}$ & $4 / 15 / 2010$ \\
\hline 2 & $\begin{array}{l}\text { Define characterization plan and schedule and } \\
\text { validate if zircaloy exists }\end{array}$ & Mark Morton & $1 / 15 / 2010$ \\
\hline 3 & $\begin{array}{l}\text { Size new building for Human Machine Interface } \\
\text { (HMI) tooling; including decontamination } \\
\text { tooling, then interface with Radcon and } \\
\text { Operations }\end{array}$ & Mark Stauder & $1 / 30 / 2010$ \\
\hline 4 & Look at bucket thumb for design application & Mark Stauder & $2 / 15 / 2010$ \\
\hline 5 & Validate IP2 self centering lid design & Colburn Kennedy & $1 / 10 / 2010$ \\
\hline 6 & $\begin{array}{l}\text { Define plan and schedule for use of shaped } \\
\text { charge in design approach }\end{array}$ & Colburn Kennedy & $1 / 20 / 2010$ \\
\hline 7 & $\begin{array}{l}\text { Provide DOE and other industry explosive } \\
\text { demolition Lessons Learned to Pat Irwin }\end{array}$ & Kim Auclair & $1 / 20 / 2010$ \\
\hline 8 & $\begin{array}{l}\text { Evaluate existing concrete data and determine } \\
\text { need for additional characterization }\end{array}$ & $\begin{array}{l}\text { Dave Lowe } \\
\text { Mark Morton }\end{array}$ & $1 / 20 / 2010$ \\
\hline
\end{tabular}




\section{APPENDIX B}

\section{EVALUATION MATRIX, MATRIX CONCLUSIONS, CRITERIA, AND ADVANTAGES \& DISADVANTAGES}




\section{WEIGHTED ANALYSIS ALTERNATIVE MATRIX}

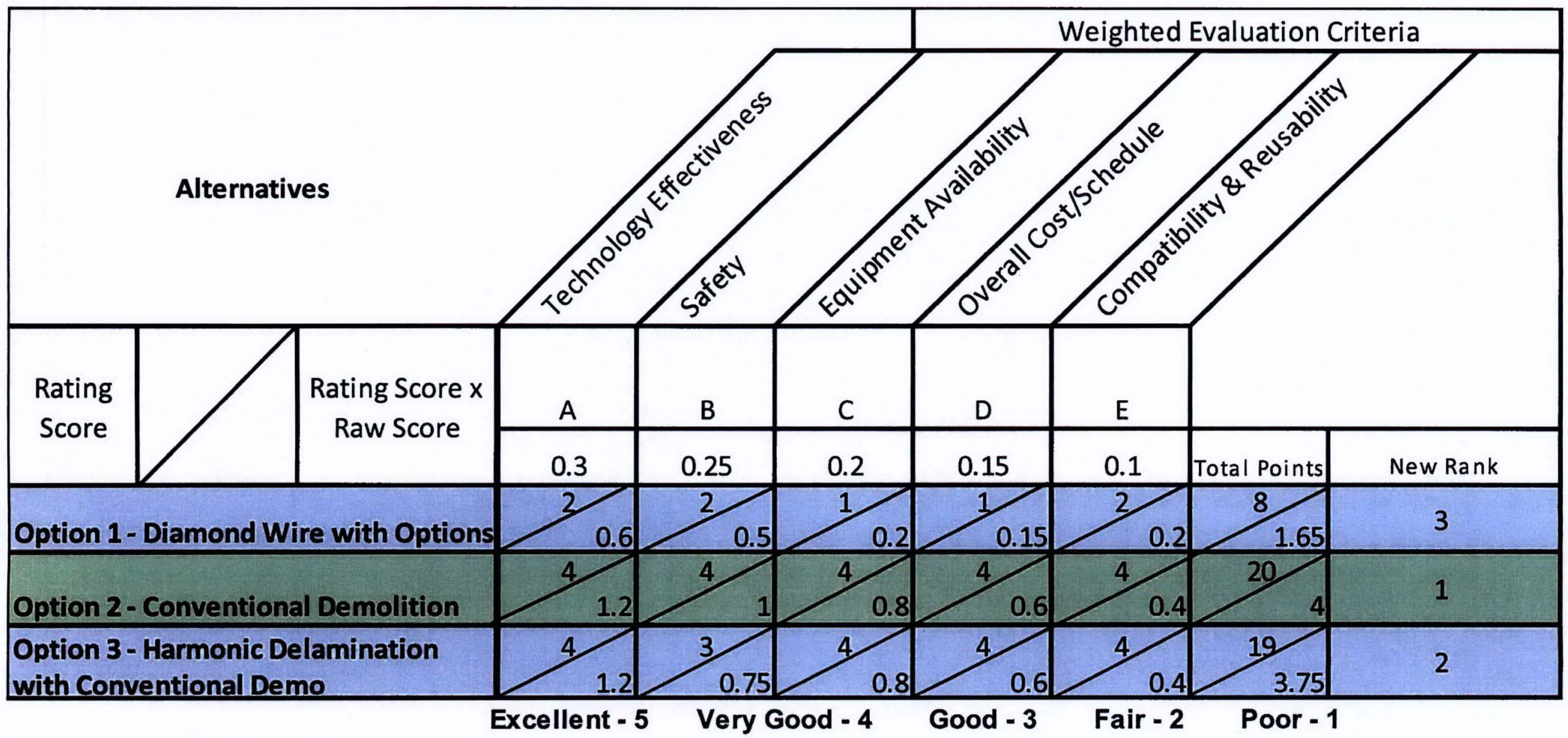




\section{EVALUATION MATRIX CONCLUSIONS}

- $\sqrt{ }$ (AI\#8) Concrete characterization would support/influence ranking on technical effectiveness, and overall cost and schedule

- Expansive grout is off-the-table

- Based on number of holes and expert advice; not the right application

- Future reactor configuration would influence all alternatives on compatibility and reusability

- Explosives is a tool, not a full option/alternative

- A tool for use with conventional demolition $>$ Similar to SAR Alternative 7

- Change title to Harmonic Delamination with Conventional Demolition 


\section{ALTERNATIVE ADVANTAGES \& DISADVANTAGES}

\section{K Reactor Core Removal Project Bio Shield Alternative Analysis}

\begin{tabular}{|c|c|c|}
\hline Rating & Evaluation Decision Risk Criteria & Percent/Value \\
\hline 1 & $\begin{array}{l}\text { TECHNOLOGY EFFECTIVENESS } \\
\text { - Technical feasibility/maturity/proven technology } \\
\text { - Ease of Operations/Ergonomics } \\
\text { - Demo duration }\end{array}$ & $30 \%$ \\
\hline 2 & $\begin{array}{l}\text { SAFETY } \\
\text { - Nuclear safety \& licensing } \\
\text { - Occupational safety } \\
\text { - Radiation safety (dose, contamination control) } \\
\text { - Fire protection }\end{array}$ & $25 \%$ \\
\hline 3 & $\begin{array}{l}\text { EQUIPMENT AVAILABILITY } \\
\text { - Reliability } \\
\text { - Robustness } \\
\text { - Failure recoverability } \\
\text { - Maintainability }\end{array}$ & $20 \%$ \\
\hline 4 & $\begin{array}{l}\text { OVERALL COST/SCHEDULE } \\
\text { - Site preparation } \\
\text { - Long lead items } \\
\text { - Installation } \\
\text { - Start-up/training } \\
\text { - Operation }\end{array}$ & $15 \%$ \\
\hline 5 & $\begin{array}{l}\text { COMPATIBILITY AND REUSABILITY } \\
\text { - Viability and execution of equipment for the entire } \\
\text { project (bio shield, thermo shield, and core removal) } \\
\text { - Future Reactor re-use }\end{array}$ & $10 \%$ \\
\hline & $\begin{array}{ll} & \text { TOTAL } \\
\end{array}$ & $100 \%$ \\
\hline
\end{tabular}




\section{ALTERNATIVE ADVANTAGES \& DISADVANTAGES}

\section{1. "Technology Effectiveness"}

\begin{tabular}{|c|c|c|c|}
\hline & (+) Advantages & & (-) Disadvantages \\
\hline & & & "Operational Challenges" \\
\hline (1) & Proven Technology (Concrete) & (1) & Unknown performance in this matrix \\
\hline 2 & Will cut the back plate & (2) & Very Time consuming \\
\hline 3 & Can begin prior to excavator installation & (3) & $\begin{array}{l}\text { Water collection and processing (i.e., new } \\
\text { waste stream) }\end{array}$ \\
\hline (4) & Regulatory buy-in & 4 & $\begin{array}{l}\text { Operational sensitivities (e.g., machine } \\
\text { binding, orientation, etc.) }\end{array}$ \\
\hline 5 & Controlled Clean Cut & 5 & $\begin{array}{l}\text { Difficulties in some obstruction (e.g., gas } \\
\text { baffles, annulus, etc.) }\end{array}$ \\
\hline 6 & $\begin{array}{l}\text { No removal of process tubes (option } \\
\text { dependent) }\end{array}$ & 6 & $\begin{array}{l}\text { Additional operation step } \\
\text { (e.g., } 25 \text { Hilti's (anchor bolts) on plate }\end{array}$ \\
\hline \multirow[t]{3}{*}{7} & No roof removal (option dependent) & & \\
\hline & \multicolumn{3}{|c|}{ Alternative 2 - Conventional Demolition } \\
\hline & (+) Advantages & & $(-)$ Disadvantages \\
\hline (1) & Proven Technology & (1) & Remote Operation \\
\hline 2 & $\begin{array}{l}\text { Initial part is non-remote } \\
\text { - Characterization dependent }\end{array}$ & 2 & Equipment (85) Modification \\
\hline (3) & Off-the-shelf tooling; simple & & \\
\hline (4) & No secondary Waste & & \\
\hline 5 & Lots of Headroom with 120 excavator & & \\
\hline 6 & No heavy lifting of bio shield blocks & & \\
\hline & Alternative & $3-$ & Explosives \\
\hline & (+) Advantages & & (-) Disadvantages \\
\hline 1 & $\begin{array}{l}\text { Concrete fracture easy to remove for } \\
\text { concrete } \\
\text { Note: Other explosives (other than } \\
\text { harmonic) are effective on steel. }\end{array}$ & (1) & Site prep time (i.e., core bores) \\
\hline 2 & Known proven approach & 2 & $\begin{array}{l}\text { Additional process step to delaminate and } \\
\text { the conventional demolition }\end{array}$ \\
\hline (3) & No lifting fixtures and block anchors & 3 & $\begin{array}{l}\text { Potential debris mitigation } \\
\text { Note: Could be part of } 4\end{array}$ \\
\hline (4) & No heavy lifting & (4) & $\begin{array}{l}\text { Over pressure event potential } \\
\text { (e.g., building and ancillary buildings } \\
\text { (adjacent or co-locate) }\end{array}$ \\
\hline
\end{tabular}




\section{ALTERNATIVE ADVANTAGES \& DISADVANTAGES}

2. "Safety"

\begin{tabular}{|c|c|c|c|}
\hline & (+) Advantages & & $(-)$ Disadvantages \\
\hline 1 & Ease of Regulatory acceptance & 1 & Handling blocks \\
\hline (2) & $\begin{array}{l}\text { Less radiation exposure } \& \text { radiological } \\
\text { hazards }\end{array}$ & (2) & High working heights \\
\hline 3 & Lower pyrophoric risk & 3 & $\begin{array}{l}\text { Handle one block at a time } \\
\text { - ERDF container }\end{array}$ \\
\hline \multirow[t]{5}{*}{ (4) } & Material handling through the tunnel & (4) & Diamond wire/rotating equipment \\
\hline & & 5 & Increased dose near the bio shield \\
\hline & & (6) & $\begin{array}{l}\text { Labor intensive } \\
\text { - Multi-discipline resources and } \\
\text { frequency; including subcontractors }\end{array}$ \\
\hline & Alternative 2-Con & venti & onal Demolition \\
\hline & (+) Advantages & & (-) Disadvantages \\
\hline 1 & Nuclear safety \& licensing & (1) & 120 metric ton excavator roof removal \\
\hline (2) & $\begin{array}{l}\text { Reduced dose } \\
\text { Note: pending surveys \& Characterization }\end{array}$ & 2 & Higher potential for contamination levels \\
\hline 3 & $\begin{array}{l}\text { Operator ease } \\
\text { No core drilling ; bang the wall }\end{array}$ & \multirow[t]{2}{*}{ (3) } & \multirow{2}{*}{$\begin{array}{l}\text { Limited robotic operations experience } \\
\text { - } 85 \& 120 \text { metric ton operator } \\
\text { experience }\end{array}$} \\
\hline $3 \mathbf{A}$ & Single process & & \\
\hline 4 & $\begin{array}{l}\text { Experienced demolition operators } \\
\text { - Including site safety culture }\end{array}$ & 4 & Flying projectiles/debris \\
\hline 5 & $\begin{array}{l}\text { No heavy lifting } \\
\text { - No blocks }\end{array}$ & \multirow{3}{*}{5} & \multirow[t]{3}{*}{ Heavy reliance on inter locks } \\
\hline 5A & No lifting/blocking features & & \\
\hline $5 \mathbf{B}$ & No elevated work & & \\
\hline 6 & $\begin{array}{l}\text { Reduced material handling } \\
\text { - Maximize loads to container }\end{array}$ & & \\
\hline \multicolumn{4}{|c|}{ Alternative 3-Explosives } \\
\hline & $(+)$ Advantages & & $(-)$ Disadvantages \\
\hline (1) & Pre-fracture/rubbilized concrete & (1) & More complicated licensing strategy \\
\hline 2 & $\begin{array}{l}\text { Standard operating procedure } \\
\text { - Simple, known process }\end{array}$ & 2 & $\begin{array}{l}\text { Elevated work } \\
\text { - Fall hazards/Stress relief fall hazards }\end{array}$ \\
\hline (3) & No heavy bio shield block lifting & (3) & More hands on work with core boring \\
\hline 4 & $\begin{array}{l}\text { Minimize industrial and radiological } \\
\text { exposures } \\
\text { - Less than Alternative } 1 \text { diamond wire }\end{array}$ & 4 & Use of explosives \\
\hline 5 & DOE experienced/available subcontractors & & \\
\hline
\end{tabular}




\section{ALTERNATIVE ADVANTAGES \& DISADVANTAGES}

3. "Equipment Availability"

\begin{tabular}{|c|c|c|c|}
\hline & (+) Advantages & & (-) Disadvantages \\
\hline (1) & $\begin{array}{l}\text { Excavator } 120 \text { no custom demolition } \\
\text { modifications or tools } \\
\text { - End effectors are the same }\end{array}$ & 1 & $\begin{array}{l}\text { More complex tool maintenance } \\
\text { (i.e., diamond saw) }\end{array}$ \\
\hline \multirow[t]{5}{*}{2} & Less excavator tool maintenance & 2 & Must core bore \\
\hline & & (3) & $\begin{array}{l}\text { Failure rate (e.g., diamond wire, hydraulic } \\
\text { excavator brakes, etc.) }\end{array}$ \\
\hline & & (4) & $\begin{array}{l}\text { Diamond wire tool (reference all four } \\
\text { criteria points) }\end{array}$ \\
\hline & \multicolumn{3}{|c|}{ Alternative 2 - Conventional Demolition } \\
\hline & (+) Advantages & & (-) Disadvantages \\
\hline 1 & $\begin{array}{l}\text { Equipment robustness } \\
\text { - Excavators }\end{array}$ & (1) & Equipment capacity of the 85 \\
\hline (2) & $\begin{array}{l}\text { Less specialized equipment } \\
\text { - No diamond core boring, etc. }\end{array}$ & 2 & $\begin{array}{l}\text { Increase excavator maintenance via work } \\
\text { on wall and shears (tool impacts) }\end{array}$ \\
\hline 3 & Bigger tools with the 120 & 3 & $\begin{array}{l}\text { Less dexterity with larger tools in smaller } \\
\text { spaces }\end{array}$ \\
\hline (4) & $\begin{array}{l}\text { Ease of equipment maintainability \& } \\
\text { spare parts }\end{array}$ & (4) & No pre-conditioned wall \\
\hline \multirow[t]{3}{*}{5} & Fewer failure scenarios & & \\
\hline & Alternat & 2 & Explosives \\
\hline & $(+)$ Advantages & & $(-)$ Disadvantages \\
\hline 1 & Simple tool and approach & 1 & $\begin{array}{l}\text { Potential explosive misfires } \\
\text { Note: this is rare }\end{array}$ \\
\hline 2 & $\begin{array}{l}\text { Less tool maintenance (i.e., excavator } \\
\text { component) }\end{array}$ & (2) & $\begin{array}{l}\text { Core drilling equipment failure recovery } \\
\text { and maintainability }\end{array}$ \\
\hline 3 & $\begin{array}{l}\text { Less tool change-out (i.e., excavator } \\
\text { component) }\end{array}$ & & \\
\hline (4) & Low failure rate & & \\
\hline
\end{tabular}




\section{ALTERNATIVE ADVANTAGES \& DISADVANTAGES}

\section{4. "Overall Cost/Schedule"

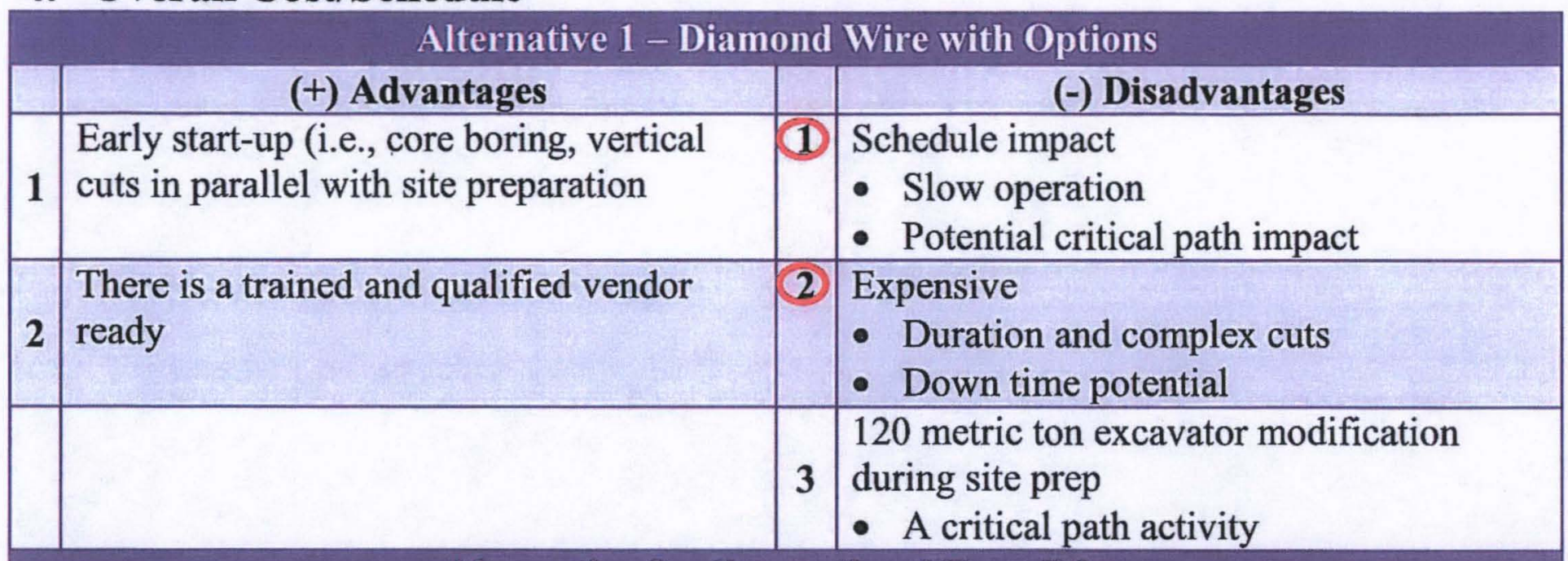

\begin{tabular}{|l|l|l|l|}
\hline \multicolumn{3}{|c|}{ Alternative 2 - Conventional Demolition } \\
\hline $\mathbf{1}$ & Continuous or single operation & & \multicolumn{1}{c|}{$(-)$ Disadvantages } \\
\hline $\mathbf{2}$ & $\begin{array}{l}\text { Minimal site prep work for the 85 metric } \\
\text { ton excavator } \\
\text { - No drilling and core boring }\end{array}$ & $\mathbf{2}$ & $\begin{array}{l}120 \text { metric ton operation site preparation } \\
120 \text { metric ton cost vs. 85 metric ton } \\
\text { excavator }\end{array}$ \\
\hline $\mathbf{3}$ & Experienced site and complex personnel & $\mathbf{3}$ & 85 metric ton excavator modification \\
\hline & & $\mathbf{4}$ & Remote operator training \\
\hline & $\mathbf{5}$ & $\begin{array}{l}\text { Longer concrete work (i.e., not pre- } \\
\text { conditioned }\end{array}$ \\
\hline
\end{tabular}

Alternative 3-Explosives

\begin{tabular}{|l|l|l|l|}
\hline \multicolumn{1}{|c|}{$(+)$ Advantages } & & \multicolumn{1}{c|}{$(-)$ Disadvantages } \\
\hline $\mathbf{1}$ & $\begin{array}{l}\text { Concrete demolition } \\
\text { - Bio shield removal }\end{array}$ & $\mathbf{1}$ & $\begin{array}{l}\text { Management reserve and schedule } \\
\text { allowance }\end{array}$ \\
\hline $\mathbf{2}$ & $\begin{array}{l}\text { * Less transportation cost with ERDF } \\
\text { containers vs. diamond wire (Alternative 1) }\end{array}$ & $\mathbf{2}$ & Public relations \\
\hline $\mathbf{3}$ & Site preparation early & $\mathbf{3}$ & Core drilling \\
\hline $\mathbf{4}$ & $\begin{array}{l}\text { Less people and processing time than } \\
\text { Alternative 1 diamond wire }\end{array}$ & $\mathbf{4}$ & $\begin{array}{l}\text { New explosive plan; environmental } \\
\text { impacts, permitting, etc. }\end{array}$ \\
\hline & & 5 & Potential impact to ongoing KW operations \\
\hline
\end{tabular}

= Significant Impact Areas

* (Same as Alternative 2 - Conventional Demolition) 


\section{ALTERNATIVE ADVANTAGES \& DISADVANTAGES}

\section{5. "Compatibility \& Reusability"}

\section{Alternative 1-Diamond Wire with Options}

(+) Advantages

(1) Major equipment pieces can be re-used

(2) Transfer Lessons Learned

Minimize tooling that is specialized

3 (e.g., shear, Hammer, etc., that is not offthe-shelf and/or special order)

Existing procedures (i.e., radcon, safety,

4 etc.) and resources; and transferable to future project
$(-)$ Disadvantages

(1) Re-mobilize diamond wire tooling options

(2) Labor intensive

Transport of large contaminated equipment

3

System alternative is only for the bio-shield

4

\section{Alternative 2 - Conventional Demolition}

(+) Advantages

1 Reduce secondary and trenchary waste

$1 A$ Least amount of consumables

2

May not need mock-ups for next project

(3) Reduce manual labor (i.e., no core boring, excavator modifications are done, etc.)

(4) Trained (resident) workforce

5 X-FR Lessons Learned

(6) Use equipment for the entire job (thermal shield, core, building, etc.)

\section{(-) Disadvantages}

(1) More wear and tear on equipment

- More maintenance

(2) 120 metric ton excavator requires building modifications on future reactors

385 excavator may require building modifications in non- $\mathrm{K}$ reactors

Mobilizing and demobilizing the 120

4 metric ton excavator-Same with Alternative 1

\section{Alternative 3 - Explosives}

(+) Advantages

Limited resources can be applied "as

1 necessary" across the job (i.e., concrete, thermal shield, etc.)

1A Works with most construction materials

(2) Public relations with successful use of explosives

3 Portable

- Can be moved easier than Alternative 1

(4) Lessons learned

- Reactor applications
(1) Public perception

(-) Disadvantages

2 Preparation time

3 Use on only the bio-shield

(4) Failure could prevent future use

- Could impact balance of work

(5) Fernald lesson: external influence limited amount of explosives - "let the experts do their job" 


\section{APPENDIX C SESSION PARKING-LOT INFORMATION SHEETS}

- Memories

- Issues/Concerns

- Assumptions

- SAR Alternative Presentation Notes

- Project Overview 
- Consider elearing pathways to acommodatolift bails; what is the minimum path

- Consideruse of gun barrels in lieu of coredrills

- $\sqrt{ }(\mathrm{AI} \# 2)$ Characterize to validate zircaloy, or not

- $\quad \sqrt{ }$ (AI\#3) Size new building (e.g., Butler type) to accommodate HMI and tooling

- $\quad \sqrt{ }(\mathrm{AI} \# 4)$ Consider use of bucket-thumb vs. clam shell, where appropriate

- $\sqrt{ }(\mathrm{AI} \# 5)$ IP2 self-centering lids

- $\quad \sqrt{ }(\mathrm{AI} \# 6)$ Consider shape charges with shear cut (ref: SAR alternative 7)

- INL-CWI Lessons Learned

- $\sqrt{ }$ Alternative 3 water jet (SAR 5) is dropped; lowest option, most expensive, cons outweigh pros. Replace with shape charges

- $\quad \sqrt{ }(\mathrm{Al} \# 3)$ Consider decontamination capabilities in the tool room

- $\sqrt{ }(\mathrm{AI} \# 7)$ Review/incorporate lessons learned across the complex

Note: Pat Irwin is working, due to complete in January 2010

$\checkmark=$ Valid memories, no action required or $\mathbf{A I}=$ Action item

\section{ISSUES/CONCERNS}

- $\sqrt{ }$ Mechanical means to remove process tubes (3200) - Solicit Kim's feedback on HMI and control room design.

Note: ongoing part of project plan.

- $\sqrt{ }$ Solicit and exchange feedback with crane operators and RCT/Radcon on HMI and control room design

Note: ongoing, part of project plan

- $\sqrt{ }$ Waste segregation recovery (Site \& ERDF)

Note: This will be a design review item

$\sqrt{ }=$ Valid issue/concern - no action required

- $\sqrt{ }$ Will not saw cut process tubes

\section{ASSUMPTIONS}

- $\sqrt{ }$ Thermal cutting techniques are allowed on outside skin of bio shield Note: Not allowed inside bio shield

- $\sqrt{\text { No method shall be used that generates zircaloy fines }}$

- $\sqrt{ }$ Process tubes, core will not be removed during site prep or obstruction removal

- $\sqrt{ }$ Pipe under work floor can be removed, as required, to install support columns

- $\sqrt{ }$ C-Elevator will be removed

$\sqrt{ }=$ Valid Assumptions 


\section{SAR ALTERNATIVE PRESENTATION NOTES}

\section{Alternative 1: Diamond Wire}

- Diamond Wire Cut (DWC) \& demolition with 85 metric ton excavator

- Core drill $\approx 42$ holes

- Install temporary shielding

- Collect/recycle and dispose coolant

- Diamond wire cuts forming 39 blocks

- During cutting process will install cut slats with temporary shielding

- Use of 85 metric ton excavator and a modified 85 metric ton excavator Note: No dose impacts until the thermal shield is exposed

- Use of two sticks; short and long to facilitate block removal to avoid interfaces

- Will remove exposed core material as practicable; demo 8' wall, then change to long stick

- Use of catch sorting tray for any debris fall out; use of bridge crane to sort and remove

- Use of standard tools: hammer, shear and clam shell bucket

- Bio shield removal will be a layered approach

- Maximize thermal shield benefit of shielding

Alternative 2: Diamond wire demolition with 120-Metric Ton Excavator

- Remove both work area cranes, install temporary scoped roof at 66' elevation; clearance for excavating arm

- Use of both 120 metric ton excavators; one is modified as appropriate

$>$ Will size reduce top blocks in sorting tray, remove exposed core material and work down the wall

$>$ Perform $\approx 44$ core drills

$>$ Use of same tools as Alternative 1: hammer, shear with larger clam-shell bucket

\section{Alternative 3: Diamond wire only and excavator}

- Remove inlet wall via cut plan

- Remove $\approx 1900$ cooling tubes/gun barrels

- Diamond wire cut forming $\approx 66$ blocks, etc.

- Install/use support system with catch net 


\section{SAR ALTERNATIVE PRESENTATION NOTES}

\section{Alternative 2 (SAR 4): Conventional Demolition}

- Full demolition of inlet face with 120 metric ton excavator

- Flame cut bio shield skin (manual operation)

- Remove/replace roof with temporary slope at 66' elevation to clear excavator arm

- * Peel wall with hammer into sorting tray

- Pierce 1" plate with hammer to allow for shear

* Layered approach or whole wall with four different tooling options

\section{Alternative 2 (SAR 8) Conventional Demolition}

- Full demolition with 85 metric ton excavator

- Use of various cutting tool options to support use of shears that have size options for the jaws

- Down select tooling via mock-up testing

\section{Alternative 3 (SAR 5): Water jet cutting}

- Water Jet with 85metric ton excavator

- Similar cut pattern to alternative 3

- Remove $\approx 1900$ gun barrels, cooling tubes, and protrusions

- Remove 750 cast iron sleeves with 8-rings on each sleeve

- Attach 66 anchor blocks (use of Hilti's)

- Water jet would not require shielding, like in alternative 1 (SAR Alternative 3)

\section{Alternative 4 (SAR 6): Expansive Grout with 85 Metric Ton Excavator}

- Flame cut; spacing with 15" (manufacturers said 12") 192 core holes and add grout

- 7-day cure time to fracture

- $\approx 9,000 \mathrm{psi}$

- Demolition similar to SAR Alternative 8, hammer, catch, load out

- Shear to size reduce back plate

\section{Alternative 7: Harmonic delamination with 85 metric ton excavator}

- Flame cut bio shield in vertical 4' sections

- Core drill on 24' diameter centers (holes)

$>26$ vertical 1.5" diameter for charges

Note: 30 ' deep

- Conventional demolition; alternative 8, including hammer and shear 


\section{SAR ALTERNATIVE PRESENTATION NOTES}

\section{SAR Summary Conclusions}

- Alternative 2 (SAR 8) was selected as the preferred alternative

- Conventional demolition with 85 metric ton excavator

- $30 \%$ design in August

- Targeted working from the top down

- Solicited CH2M and other SME's around the country

New Alternative: Shaped Charges with shear cut (see SAR Alternative 7)

- Surgical demolition with specialized explosives and highly trained people

- INL-CWI Lessons with smaller reactor

- Use TNT down a hole to blow away from the reactor. Had $\approx 2$ ' thicker bio shield

- Combine with delamination Alternative 4 and SAR Alternative 7

Note: See page 48 of SAR design report

\section{PROJECT OVERVIEW}

- Core characterization will be done late January/early February of 2010

- Detectors will be on all remote equipment

- Overview obstruction removal and base-line assumptions

- Will be collecting dose rates over the next two weeks

- Currently conducting a graphite literature search as well

- Fixatives will be applied wherever it can be reached 
CHPRC-00573

Revision 0

\section{APPENDIX D}

\section{WORKSHOP AGENDA, GUIDELINES AND EXPECTATIONS, OPENING REMARKS, AND ATTENDANCE ROSTER}


CHPRC-00573

Revision 0

\section{KE REACTOR CORE REMOVAL PROJECT \\ ALTERNATIVE ANALYSIS WORKSHOP \\ WSU-CIC, Conference Room 214 \\ December 15-16, 2009}

Purpose: $\quad$ Select the preferred Bio Shield Alternative, for integration with the Thermal Shield and Core Removal and develop the path forward to proceed with project delivery

- Overview Project Scope and Bio Shield Alternative purpose and needs

- Utilize weighted analysis criteria to select the preferred Bio Shield Alternative

- Develop path forward actions required to proceed

\section{AGENDA}

Day 1, Tuesday, December 15, 2009

7:30 - Welcome/Purpose, Safety Topic, and Introductions

- Review Agenda, Guidelines and Expectations

- Opening Remarks (Objectives and Success)

8:00 - Conduct Project and Bio Shield Alternative Presentations

- Project Scope Layout and Approach - Mark Morton

- Present Bio Shield Alternatives

1. Diamond Wire and Options (SAR Options 1,2\&3) - Mark Stauder

- Utilize parking-lot sheets, as appropriate

9:15 - BREAK

9:30 - Continue Project Presentations

- Present Bio Shield Alternatives

2. Conventional Demolition (SAR Options 4\&8) - $\quad$ Mark Stauder

3. Expansive Grout (SAR Options 5) - $\quad$ Mark Stauder

4. Explosives, Expansive Grout (SAR Options 6\&7) - $\quad$ Mark Stauder

- Utilize parking-lot sheets, as appropriate

11:45 - WORKING LUNCH

12:30 - Overview Evaluation Criteria \& Develop Advantages \& Disadvantages

- Review/clarify weighted evaluation criteria

- Define alternative advantages and disadvantages by criteria

- Utilize parking-lot sheets, as appropriate

2:30 - BREAK

2:45 - Complete Definition of Advantages \& Disadvantages

- Utilize parking-lot sheets, as appropriate

4:30 - Finish day 1 with review of status and day 2 agenda 
CHPRC-00573

Revision 0

\section{KE REACTOR CORE REMOVAL PROJECT ALTERNATIVE ANALYSIS WORKSHOP \\ WSU-CIC, Conference Room 214 \\ December 15-16, 2009}

Purpose: Select the preferred Bio Shield Alternative, for integration with the Thermal Shield and Core Removal and develop the path forward to proceed with project delivery

- Overview Project Scope and Bio Shield Alternative purpose and needs

- Utilize weighted analysis criteria to select the preferred Bio Shield Alternative

- Develop path forward actions required to proceed

\section{AGENDA}

Day 2, Wednesday, December 16, 2009

7:15 - Review Agenda, Status, and Safety Topic

7:30 - Conduct Weighted Analysis Matrix

- Apply weighted criteria and rate alternatives

- Utilize parking-lot sheets, as appropriate

9:30 - BREAK

9:45 - Develop Path Forward Implementation Plan

- Review/validate parking-lot information sheets

- Define next steps and actions required to proceed

11:00 - Finish Session with a Round Robin Close-Out

- Last minute items

- Meeting utility and closing remarks 


\section{GUIDELINES \& EXPECTATIONS}

- Open and honest communication

- Active listening

- Courage \& consideration

- Be succinct $\rightarrow$ Make your point

- Lots of ground to cover

- Identify facts vs. perceptions $>$ Clarify as required

- Obstruction removal alternative analysis

- Bioshield: Removal inlet face to support/allow core removal; $>$ Includes thermal shield and core removal work/activities

- SAR report: eight alternatives that will be condensed to four alternatives and ranked by the decision board

- Use of flipcharts and infocus

- For all to see and document

- Will be issued in the workshop report

- Keys to Success

- Communication and teamwork $>$ Support to decision board to evaluation and rank

- Focus on next steps to support design and project delivery

\section{OPENING REMARKS}

- Thanks for your active participation on the first project of this type at Hanford

- Lots of Experience

$>$ Including SME's (Dave Lowe, Dan Coyne and Denny Ferrera) from multiple DOE Sites (Idaho, Rocky Flats, Mound, etc.)

- We have a good craft cross section and SME's on this project

- Look forward to the skill of the craft and the participation

- First step in getting this core out safely and economically

- Define and implement administrative/hazard controls 


\begin{tabular}{|c|c|c|c|c|}
\hline \multicolumn{5}{|c|}{$\begin{array}{c}\text { 100KE Reactor Core Removal Project Alternative Analysis Workshop } \\
\text { Attendance Roster } \\
\text { December 15-16, 2009 }\end{array}$} \\
\hline Name & Phone & Organization & 12/15/09 & $12 / 16 / 09$ \\
\hline Dave Lowe & $303-994-4058$ & CH2MHILL & $\mathrm{X}$ & $\mathrm{X}$ \\
\hline Darren Boone & $509-373-0891$ & CHPRC & $\mathrm{X}$ & $\mathrm{X}$ \\
\hline James Seitz & $509-942-3952$ & $\overline{\text { CHPRC }}$ & $\mathrm{X}$ & $\mathrm{X}$ \\
\hline Ellen Dagan & $509-376-3811$ & DOE-KBC & $\mathrm{X}$ & $\mathrm{X}$ \\
\hline Dan Coyne & $208-351-9867$ & CWI-ICP & $\mathrm{X}$ & $\mathrm{X}$ \\
\hline J. Mike Swartz & $509-727-8767$ & CHPRC & $\mathrm{X}$ & $\mathrm{X}$ \\
\hline Bill Linzau & $509-373-0101$ & DNFSB & $\mathrm{X}$ & \\
\hline Denny Ferrera & $303-330-9323$ & CH2MHILL & $\mathrm{X}$ & $\mathrm{X}$ \\
\hline Colburn Kennedy & $509-531-5300$ & $100 \mathrm{~K}$ & $\mathrm{X}$ & $\mathrm{X}$ \\
\hline Rick Bond & $509-372-7885$ & Ecology & $\mathrm{X}$ & \\
\hline Jason Pearson & $509-492-0148$ & MSA & $\mathrm{X}$ & $\mathrm{X}$ \\
\hline Bill Kirby & $970-593-2153$ & SAR & $\mathrm{X}$ & $\mathrm{X}$ \\
\hline Eric Eller & $970-690-0479$ & SAR & $\mathrm{X}$ & $\mathrm{X}$ \\
\hline Mark Stauder & $970-481-4686$ & SAR & $\mathrm{X}$ & $\mathrm{X}$ \\
\hline Kim Auclair & $360-609-0627$ & Lucas & $\mathrm{X}$ & $\mathrm{X}$ \\
\hline Robert Norris & $509-850-6961$ & SAR & $\mathrm{X}$ & $\mathrm{X}$ \\
\hline Michael Koch & $509-627-0783$ & CHPRC/100K Engineering & $\mathrm{X}$ & \\
\hline Mark Morton & $509-727-2929$ & Worley Parsons & $\mathrm{X}$ & $\mathrm{X}$ \\
\hline Brenda Pangborn & $509-372-3841$ & DOE-RL RCM & $\bar{X}$ & $\bar{X}$ \\
\hline Don Foti & $509-373-6090$ & CHPRC & $\mathrm{X}$ & $\mathrm{X}$ \\
\hline Burt Hill & $509-376-6863$ & DOE-RL & $\mathrm{X}$ & \\
\hline Greg Morgan & $509-373-2346$ & DOE-RL & $\mathrm{X}$ & \\
\hline Rod Lobos & $509-376-3749$ & EPA & $\mathrm{X}$ & $\mathrm{X}$ \\
\hline Calvin Morgan & $509-376-5018$ & CHPRC SHS\&Q & $\mathrm{X}$ & $\mathrm{X}$ \\
\hline Tom Teynor & $509-376-6363$ & DOE-RL & $\mathrm{X}$ & $\mathrm{X}$ \\
\hline Marty Plys & 312-953-7299 & Fauske & $\mathrm{X}$ & \\
\hline
\end{tabular}

